\title{
Tinnitus, CTCAE 5.0
}

National Cancer Institute

\section{Source}

National Cancer Institute. Tinnitus, CT CAE 5.0. NCI Thesaurus. Code C146690.

A disorder characterized by noise in the ears, such as ringing, buzzing, roaring or clicking. 